Article

\title{
Elovanoid 34 reduces proinflammatory cytokines and arrest the expression of genes that drive senescence, autophagy, unfolded protein response (UPR), matrix metalloproteinases (MMPs) and inflammation in human nasal cells exposed to house dust mite (HDM): Therapeutic Potential in Allergy
}

\author{
Alfredo Resano1, Miguel Barajas ${ }^{1, *}$, Surjyadipta Bhattacharjee ${ }^{3}$, Roberto Aguado-Jiménez ${ }^{1}$, David Rodríguez², Ri- \\ cardo Palacios², Nicolás G Bazán ${ }^{3, *}$ \\ 1 Department of Health Science. Public University of Navarra. Pamplona. Spain; alfredo.resano@unavarra.es \\ (AR); miguel.barajas@unavarra.es (MB); roberto.aguado@unavarra.es (RA) \\ 2 Diater Laboratorio. Madrid. Spain; d.rodriguez@diater.com (DR); r.palacios@diater.com (RP) \\ 3 Neuroscience Center of Excellence. Louisiana State University (LSUHSC). New Orleans. US; bhattachar- \\ jee@lsuhsc.edu (SB); nbazan@lsuhsc.edu (NB) \\ * Correspondence: miguel.barajas@unavarra.es (MB) \& nbazan@lsuhsc.edu (NB).
}

\begin{abstract}
Elovanoids (ELV) are novel lipid mediators synthesized from very long-chain (VLC) omega-3 (n-3) polyunsaturated fatty acids (PUFA), particularly docosahexaenoic acid (DHA) or eicosapentaenoic acid (EPA), by the elongase enzyme ELOVL4. ELV have been shown to play a fundamental role in maintaining brain and retina function, neuroprotection, maintaining the skin's permeability barrier, and sperm function, among other important cellular processes. Furthermore, the methyl ester or sodium salt of ELV-N32 and ELV-N34 have been found to be efficient in reduced infarct volumes, promoting cell survival and decreasing the alteration of the neurovascular unit in a model of ischemia due to occlusion of the middle cerebral artery. Their bioactivity includes enhancing of anti-apoptotic and pro-survival protein expression combined with the downregulation of pro-apoptotic proteins. House dust mite (HDM) is the most prevalent indoor allergen, and HDM exposure is frequently the cause of allergic respiratory disease, including allergic rhinitis and allergic asthma in HDM sensitized individuals. In our study, human nasal epithelial cells (HNEpC) were used as an in vitro model for allergy. The cells were challenged with HDM (mixture of Dermatophagoides farinae and D. pteronyssinus extract), with or without treatment with ELV-34. Phosphate-buffered saline (PBS) (vehicle) and ELV-34 (500 nM) alone were used as the control. Our findings show that ELV-34 promotes cell viability and reduces cytotoxicity upon HDM sensitization of HNEpC. ELV-34 treatment significantly reduces the levels of IL-6, IL-1 $\beta$, IL-8, VEGF, ICAM1, CXCL1 and CCL2, restored the IL-10 level and diminishes the induction of the transcription of inflammatory and senescence genes in HNEpC. Our data also shows that HDM triggered the expression of several key genes in human nasal epithelial cells, including autophagy, unfolded protein response (UPR), Matrix metalloproteinases (MMPs), senescence and inflammation markers. Together, our data reveal a novel pro-homeostatic and cell-protective lipid-signaling mechanism in nasal epithelial cells that envision novel potential therapeutic targets for the treatment of allergies.
\end{abstract}

Keywords: elovanoid; allergy; house dust mite, HNEpC.

\section{Introduction}

Elovanoids are a bioactive lipid mediators derived from VLC-PUFA, n-3, which are the biosynthetic products of elongase ELOVL4 newly-discovered that are necessary for 
cell integrity and have shown very promising results in a cellular experimental allergy model in human nasal cells exposed to HDM. These novel lipid mediators have been shown to be able to downregulate senescence gene programming, SASP (senescence-associated secretory phenotype), and inflammaging (age-dependent low-grade chronic inflammation) which in turn, promotes and sustains cell structural integrity, viability, and function $[10,14]$.

House dust mites (HDM) are the most important indoor allergen, approximately 1$2 \%$ of the global population suffers from house dust mite allergy.is the single most important domestic source for allergic diseases such as allergic rhinitis, asthma and atopic dermatitis. The most important house dust mites are Dermatophagoides pteronyssinus and Dermatophagoides farinae (in drier areas) and Euroglyphus maynei (in humid areas). HDM allergen plays important role in allergic inflammation through innate immune system Toll-like receptors (TLRs), protease activated receptors (PARs) and DC-SIGN could be activated by different HDM major allergens, resulting in the upregulation of proinflammatory cytokines.

Because Elovanoids have been shown to play an important role in the control of mediators involved in inflammation, exerting a protective role of cellular integrity against inflammation[15], we proposed to determine whether this property could be useful to protect a human nasal epithelial cell line (HNEpC) exposed to HDM-induced deleterious action.

Some serum markers of inflammation, for example IL-6, commonly tend to be slightly higher in older adults than the usual baseline in younger adults, it has been proposed that this causes damage to normal tissues and contributes to degenerative diseases of aging or inflammaging phenomen. The inflammaging is partly attributable to dysregulated immune system leading to the development of the SASP program consistent of the release of multiple cytokines and chemokines by accumulation of senescent nonimmune tissue cell with a proinflammatory effect. The dysregulation, not only in SASP program, but also in the processes of autophagy and mitophagy, have been associated with environmental allergen or pollulant [16].

\section{Results}

\subsection{Elovanoids elicit potent cytoprotection against LPS, poly(I:C) and HDM extracts.}

To test the efficacy of elovanoids on cell survival, human nasal epithelial cells HNEpC were incubated with LPS $(30 \mu \mathrm{g} / \mathrm{mL})$ or poly(I:C) $(100 \mu \mathrm{g} / \mathrm{mL})$ and 30 minutes later ELVN32 and ELVN-34 (500nM) were added for 24h hours (Figure 1).

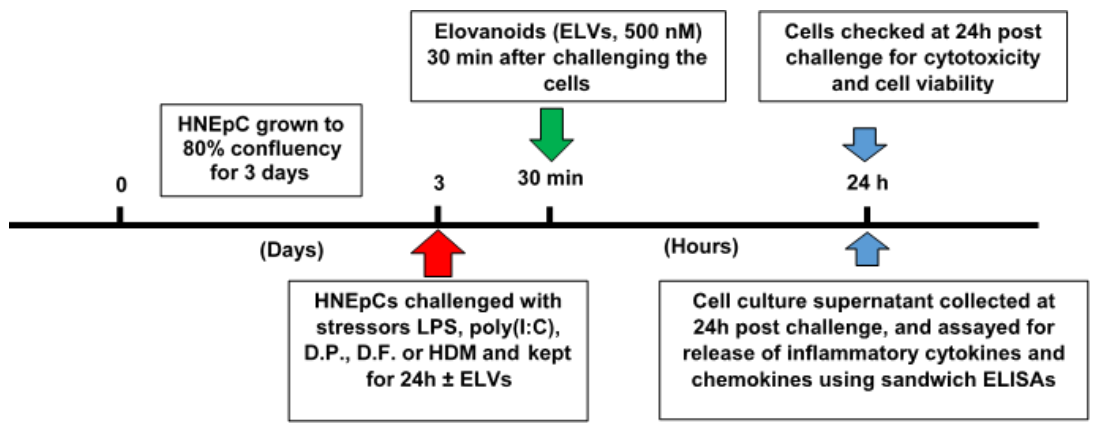

Figure 1. In vitro experimental design used in the study. Human nasal epithelial cells (HNEpC) challenged by allergen HDM were analyzed to determine the protective effect of Elovanoids.

LPS and poly(I:C) induced a decrease in cell viability and increase of LDH levels (Figure 2 , red bars), indicating that $\mathrm{HNEpC}$ were are subjected to cellular stress that compromises their viability. Our results indicate that both ELVN-32 and ELVN-34 successfully prevented cell death, restoring the cell viability (Presto Blue HS assay) (Figure 2, left panel) 
and LDH levels (cytotoxicity assay, CyQuant LDH) (Figure 2, right panel) in all the conditions tested. Addition of ELVs increases cell viability and fosters protection to the HNEpC.

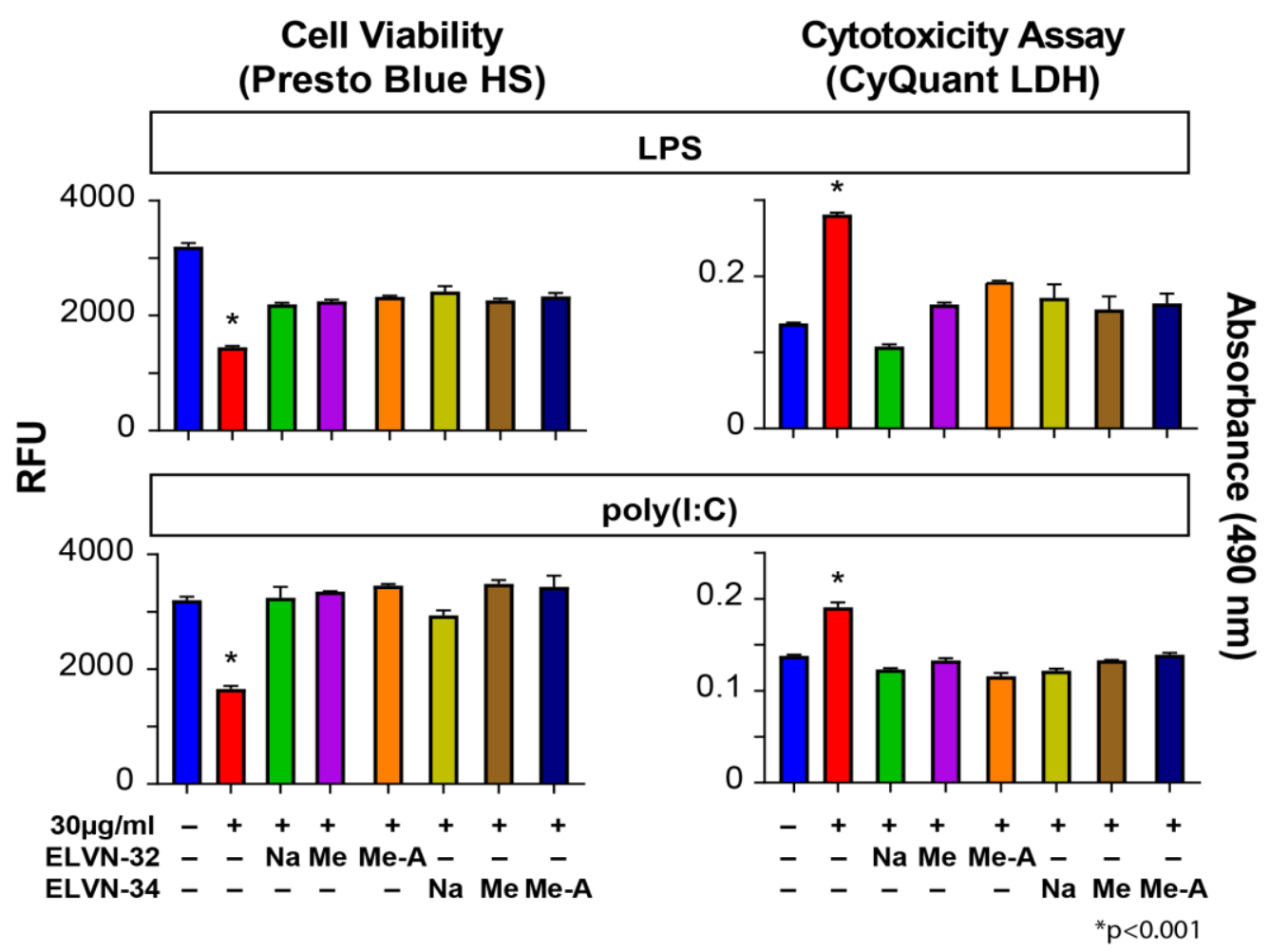

Figure 2. ELVN-32 and ELVN-34 protect HNEpC from cytotoxicity induced by LPS or poly(I:C). Cytotoxicity assay (CyQuant LDH, right panels) shows that ELVN-32 and ELVN-34 elicit potent HNEpC cytoprotection when are exposed to the stressors LPS (red bars, upper panel) and poly(I:C) (red bars, botton panel). HNEpC treated with ELVN-32 and ELVN-34 (500nM) were protected from the cell death (Presto Blue HS, left panels) induced by LPS $(30 \mu \mathrm{g} / \mathrm{mL})$ or poly(I:C) $(100 \mu \mathrm{g} / \mathrm{mL})$. The results showed the averages of three independent experiments. ${ }^{*} \mathrm{p}<0.001$

Asthma is caused by exposure to allergens such as dust mites, pet dander, pollen, or mold. Of these allergens, house dust mite (HDM) is the most important indoor allergen for humans, although two other mite species, Dermatophagoides pteronyssinus (D. pteronyssinus) and Dermatophagoides farinae (D. farinae), have been also shown to play a relevant role in the development of asthma in human patients.

HDM extracts (D. farinae, D. pteronyssinus and HDM) induced a decrease in cell viability (Figure 3, red bars at left panel) and increase of LDH levels (Figure 3, red bars at right panel), indicating that HNEpC were are subjected to cellular stress that compromises their viability.

Cytotoxicity assay (LDH) shows that the stressor HDM extracts (D. farinae, D. pteronyssi$n u s$ and HDM) increase formation of red formazan indicating cytotoxicity, which is reduced by ELVs (Figure 3, right panel). Cell viability assay using PrestoBlue HS reagent also shows more resorufin production to cells challenged with the stressor HDM extracts (D. farinae, D. pteronyssinus and HDM) (Figure 3, left panel). 

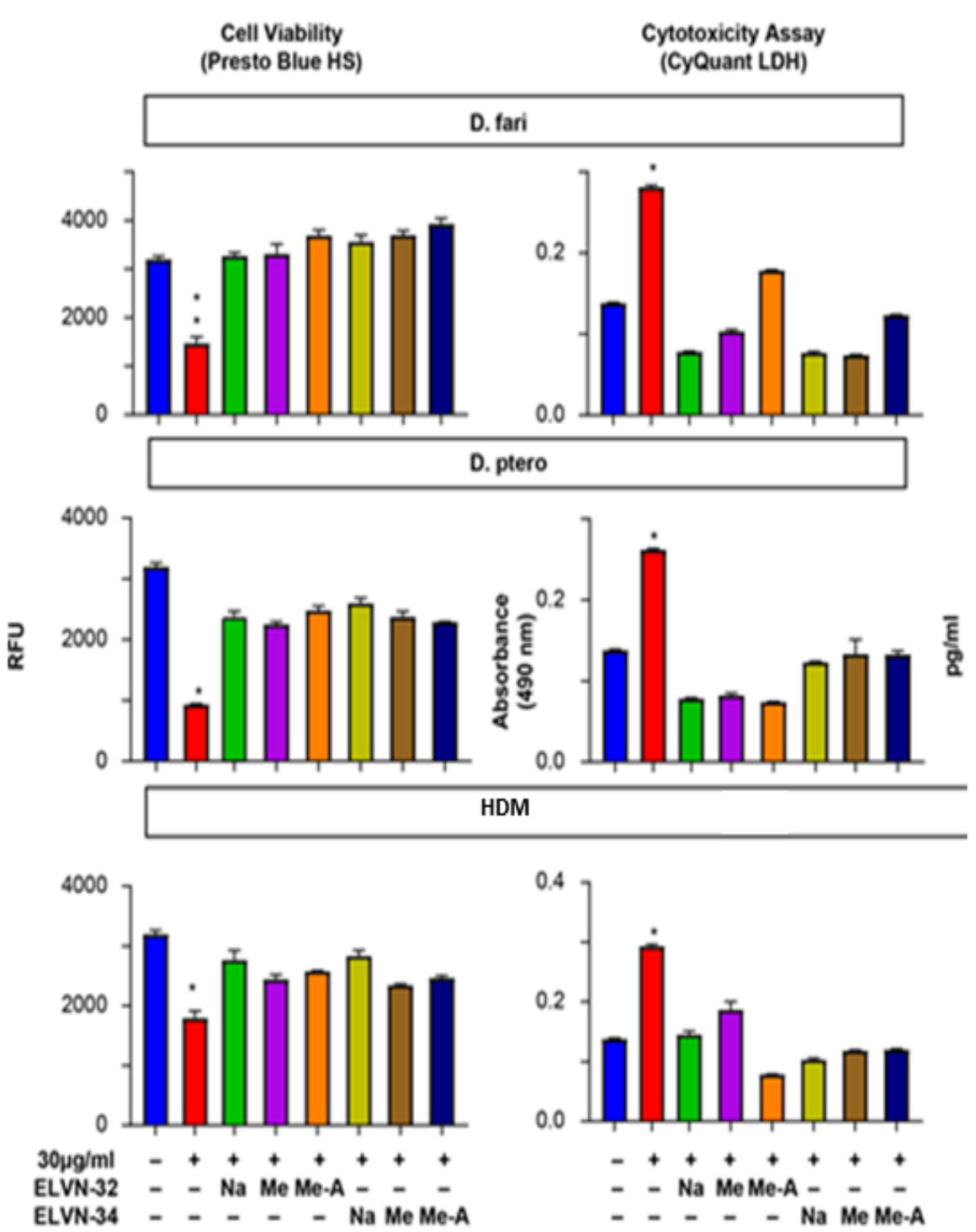

Figure 3. ELVN-32 and ELVN-34 protect HNEpC from cytotoxicity induced by HDM extracts. Cytotoxicity assay (CyQuant LDH, right panels) shows that ELVN-32 and ELVN34 elicit potent HNEpC cytoprotection when are exposed to the stressors HDM extracts (Dermatophagoides farinae, Dermatophagoides pteronyssinus and HDM). HNEpC treated with ELVN-32 and ELVN-34 (500nM) were protected from the cell death (Presto Blue HS, left panels) induced by HDM extracts. The results showed the averages of three independent experiments. ${ }^{*} \mathrm{p}<0.001$

Our results indicate that both ELVN-32 and ELVN-34 successfully prevented cell death, restoring the cell viability (Presto Blue HS assay) (Figure 3, left panel) and LDH levels (cytotoxicity assay, CyQuant LDH) (Figure 3, right panel) after the challenge with different HDM extracts. In summary, addition of ELVs increases cell viability as well as HNEpC protection.

2.2 Elovanoids downregulate the expression of pro-inflammatory cytokines and chemokines induced by LPS, poly(I:C) and HDM extracts in human epithelial nasal cells. 
HNEpC were exposed 30 minutes to LPS $(30 \mu \mathrm{g} / \mathrm{mL})$ or poly(I:C) $(100 \mu \mathrm{g} / \mathrm{mL})$ and their supernatants were collected in order to determine the expression of pro-inflammatory cytokines and chemokines IL-6, IL-1 $\beta$, IL-8/CXCL8, CCL2/MCP-1, CXCL1/KC/GRO, VEGF, ICAM1(CD54). Our results demonstrate that the challenge of HNEpC with LPS or poly(I:C) induce the expression of pro-inflammatory cytokines and chemokines IL-6, IL$1 \beta$, IL-8/CXCL8, CCL2/MCP-1, CXCL1/KC/GRO, VEGF, ICAM1(CD54) (Figure 4, red bars). The treatment of human nasal epithelial cells with ELVN-32 (500nM) or ELVN-34 $(500 \mathrm{nM})$ reduce the expression of pro-inflammatory cytokines and chemokines (Figure 4 ). However, LPS downregulates the expression IL-10 in HNEpC that is restored to normal levels after treatment with ELVN-32 or ELVN-34 (Figure 5, panel IL-10). These results show that treatment with elovanoids protects human nasal epithelial cells from the expression of pro-inflammatory cytokines under the stressors LPS and poly(I:C), favoring the expression of anti-inflammatory cytokines such as IL-10.
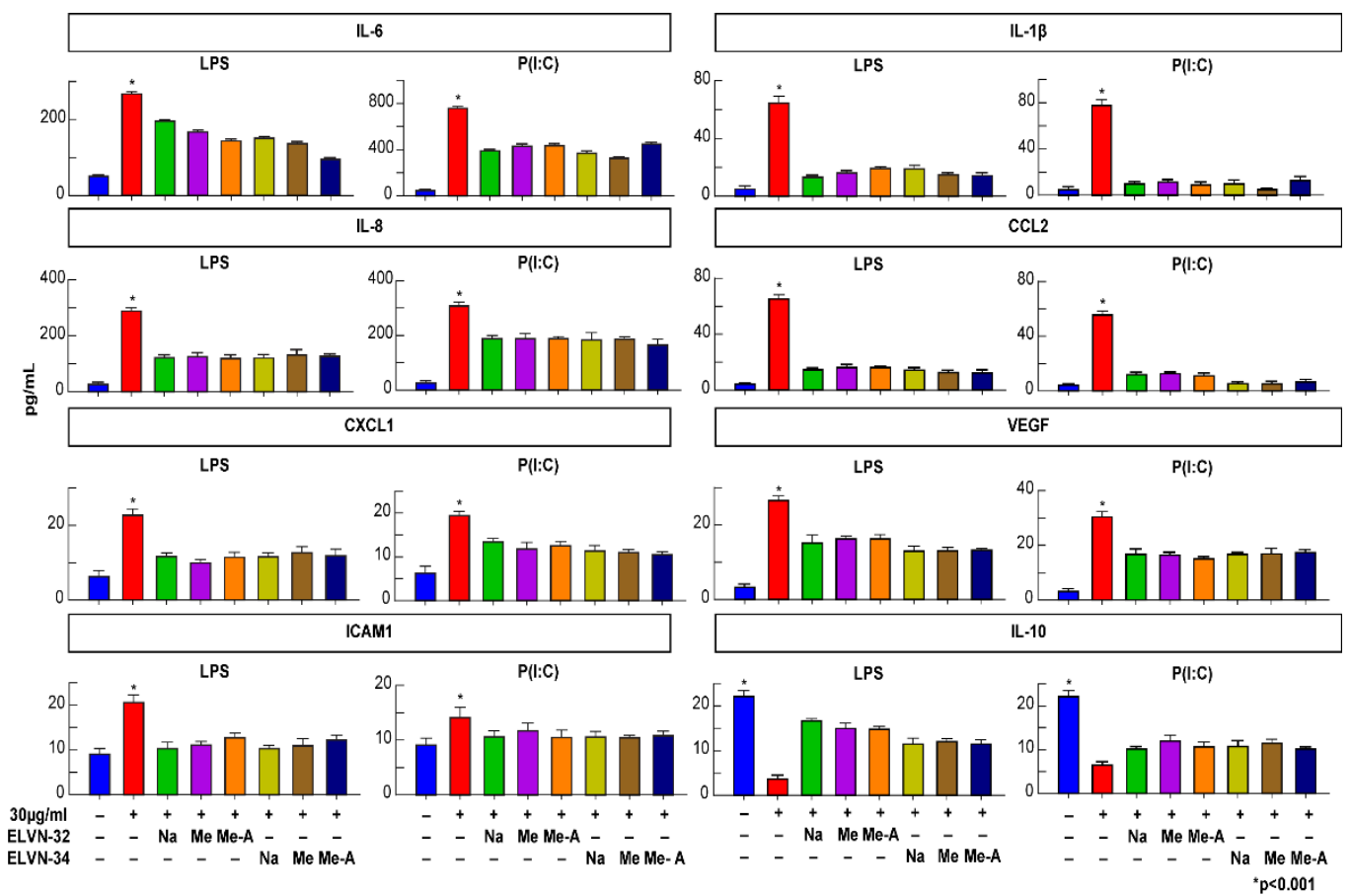

Figure 4. ELVN-32 and ELVN-34 reduce the expression of pro-inflammatory cytokines and chemokines in HNEpC treated with LPS or poly(I:C). HNEpC challenged with LPS $(30 \mu \mathrm{g} / \mathrm{mL})$ or poly(I:C) $(100 \mu \mathrm{g} / \mathrm{mL})$ display enhanced production of pro-inflammatory cytokines and chemokines IL-6, IL-1 $\beta$, IL-8/CXCL8, CCL2/MCP-1, CXCL1/KC/GRO, VEGF, ICAM1(CD54) compared to non-treated cells. This increase in the production of pro-inflammatory cytokines and chemokines is abrogated by the addition of ELVN-32 or ELVN-34 $(500 \mathrm{nM}) 30$ minutes post-challenge. LPS induces a reduction of IL-10 expression in HNEpC that is restored to normal levels after treatment with ELVN-32 or ELVN-34. The results showed the averages of three independent experiments. ${ }^{*} \mathrm{p}<0.001$

Similar results were observed when HNEpC were challenged with HDM stressors ( $D$. farinae, D. pteronyssinus and HDM) $(30 \mu \mathrm{g} / \mathrm{mL})$ (Figure 5, red bars). The treatment of human nasal epithelial cells with ELVN-32 (500nM) or ELVN-34 (500nM) reduce the expression of pro-inflammatory cytokines and chemokines induced by HDM stressors (Figure 5). Again, HDM stressors showed a significant downregulation of the IL-10 expression in HNEpC that is restored to normal levels after treatment with ELVN-32 or ELVN-34 (Figure 5, panel IL-10).

These results indicate that treatment with elovanoids protects human nasal epithelial cells from the expression of pro-inflammatory cytokines, in favor of anti-inflammatory cytokines, suggesting a possible beneficial therapeutic effect for the treatment of allergies. 

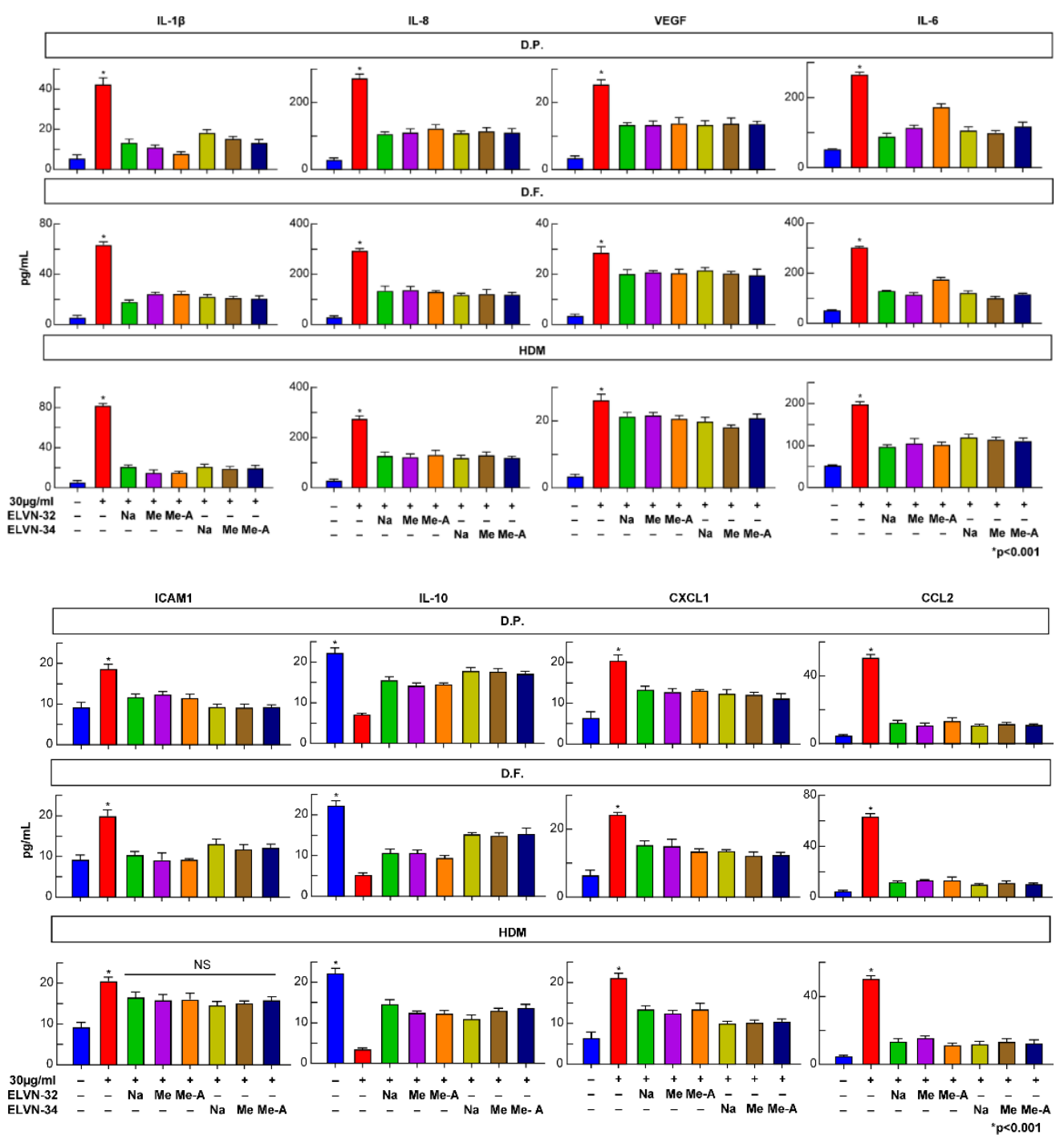

Figure 5. ELVN-32 and ELVN-34 reduce the expression of pro-inflammatory cytokines and chemokines in HNEpC exposed to HDM extracts. A remarkable decrease was observed in the release of anti-inflammatory cytokine IL-10 in HNEpC challenged with the HDM stressors (D. farinae, D. pteronyssinus and HDM) $(30 \mu \mathrm{g} / \mathrm{mL})$. This decreased production of anti-inflammatory cytokine are reversed by the addition of ELVN-32 or ELVN$34(500 \mathrm{nM}), 30$ minutes post challenge with the stressor. The results showed the averages of three independent experiments. ${ }^{*} \mathrm{p}<0.001$

2.3 Elovanoids regulate the expression of autophagy, UPR, MMPs, senescence and inflammation markers in human epithelial nasal cells.

To search for the mechanism involved in ELVs protection against HDM-mediated damage, UPR, MMPs, senescence and inflammation markers were analyzed by quantitative PCR on HNEpC challenged with HDM stressors (D. farinae, D. pteronyssinus and HDM) $(30 \mu \mathrm{g} / \mathrm{mL}$ ). We selected genes involved in autophagy (ATG3, ATG5, ATG7, BECLIN-1 and P62), unfolded protein response (UPR) (ATF6, CHOP and IRE1) and matrix metalloproteinases (MMPs) genes (MMP8, MMP2, MMP9, MMP3, MMP12, TIMP12 and TIMP2) (Figure 6A), as well as, the expression of senescence (P21, P16, P27 and P53) and inflammation genes (IL-1 $\alpha$, IL-6 and IL-1 $\beta$ ) (Figure 6B). All the markers analyzed showed high expression in HNEpC after HDM treatment, with the exception of p53 (Figure 6, red bars). The treatment of human nasal epithelial cells with ELVN-32 (500nM) or ELVN-34 (500nM) 
significantly reduce the expression of all genes mentioned above after challenge with HDM stressors (Figure 6, blue bars).

These results clearly indicate that elovanoids have a protective effect on the survival, integrity, senescence and inflammation, as well as, autophagy process at the cellular level.

A.
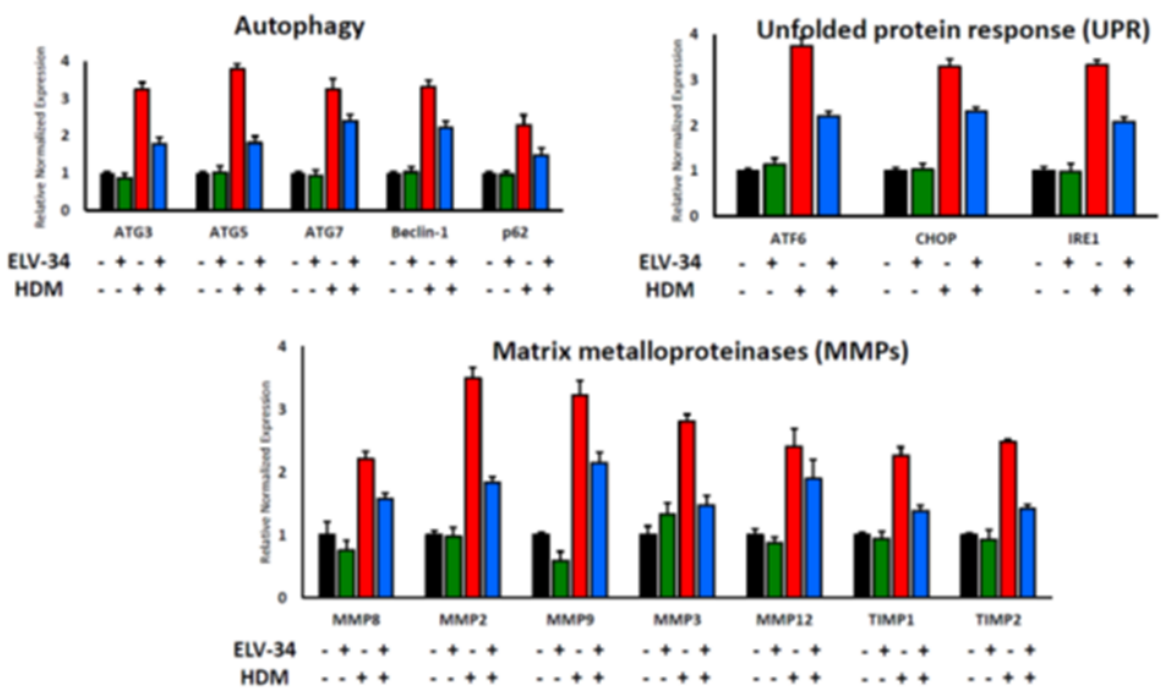

B.
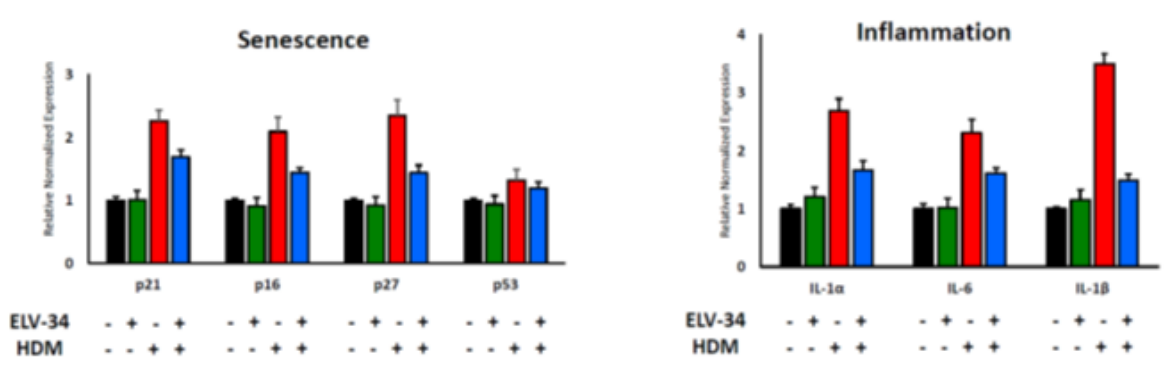

Figure 6. HDM triggered multiple signaling in human nasal epithelial cells. The challenge of HNEpC with HDM stressors (D. farinae, D. pteronyssinus and HDM) $(30 \mu \mathrm{g} / \mathrm{mL})$ induce the expression of autophagy (ATG3, ATG5, ATG7, BECLIN-1 and P62), unfolded protein response (UPR) (ATF6, CHOP and IRE1) and matrix metalloproteinases (MMPs) genes (MMP8, MMP2, MMP9, MMP3, MMP12, TIMP12 and TIMP2) (panel A). HDM stressors also induce the expression of senescence (P21, P16, P27 and P53) and inflammation genes (IL-1 $\alpha$, IL-6 and IL-1 $\beta$ ) on HNEpC (panel B). The treatment with ELVN-34 (500 nM) reduce the expression of autophagy, UPR, MMPs, senescence (except P53) and inflammation genes induced by HDM extracts. The results showed the averages of three independent experiments. ${ }^{*} \mathrm{p}<0.001$

\subsection{Elovanoids protect human nasal epithelial cells from senescence.}

Finally, our study also evaluated the Senescence-Associated Secretory Phenotype (SASP response) in human nasal epithelial cells (HNEpC), finding that treatment with elovanoids protected these cells from this phenomenon. HDM extract $(30 \mu \mathrm{g} / \mathrm{mL})$ induced an increased number of beta-galactosidase positive cells (Figure 7, red bar) indicating that cellular senescence has been activated as a consequence of the stressor used. ELVN-34 $(500 \mathrm{nM}$ ) elicit a robust protection of HNEpC exposed to HDM extract (Figure 7, green 
bar), suggesting that this novel compound could protect HNEpC from premature senescence induced by the exposure to allergens.

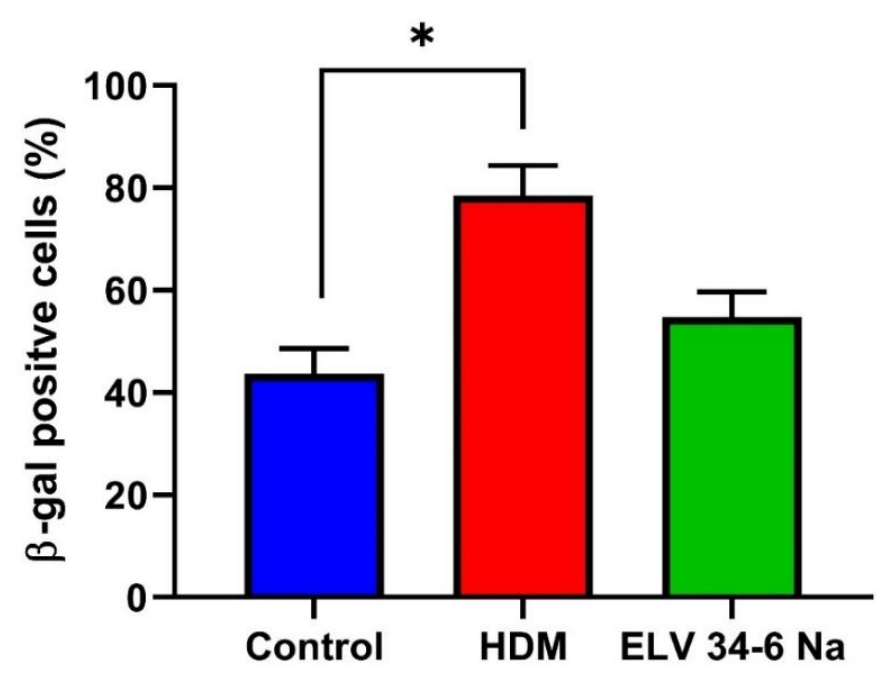

Figure 7. ELVN-34 protect HNEpC from the senescence-associated secretory phenotype (SASP response). Quantitative assessment of human nasal cells increased the SASP response (measured by the spider $\beta$-galactosidase staining) upon HDM exposure (30 $\mu \mathrm{g} / \mathrm{mL})$. ELV 34:6 (500nM) treatment reduces the number beta-gal positive HNEpC induced by HDM. The results showed the averages of three independent experiments. ${ }^{*} \mathrm{p}<0.001$

\section{Discussion}

In the present study we use a model of human nasal epithelial cells (HNEpC) to study the release of inflammatory cytokines and the expression of adhesion molecules when interacting with already known inflammatory inducers, LPS and Poly (I: C), as well as when reproducing the in vivo conditions of HDM allergen-stimulated allergic rhinitis. Both cytotoxicity (damage to the plasma cell membrane) and cell viability (metabolic activity of cells) have been altered with exposure to these known inflammatory inducers of innate immunity LPS [17] and Poly (I: C) [18], as well as against house dust mites Dermatophagoides pteronyssinus, Dermatophagoides farinae or a 50\% mixture of both (HDM).

The high sensitization capacity of HDM is known by inducing the proliferation of Th0 to Th2 lymphocytes, limiting the differentiation to Th1, and it has been attributed to the ability of group 1 allergens (Der p1), for example, to cleave IL-2R [19], activate dendritic cells for Th2 polarization, suppress IL- 12 by CD40 cleavage, reduce INF- $\gamma$ release and increase IL-4 production [20], thus favoring an allergic microenvironment by acting on adaptive immunity. But there is growing evidence that these group 1 allergens are mainly responsible for activating the innate immune response, cleaving the binding proteins of epithelial cells, activating protease-sensitive receptors (PAR) and TLR4, activating NOD-like receptors. (NLR) or interacting with ILC [21]. Wong et al. [22] studied the effects of HDM Dermatophagoides pteronyssinus [1] on the activation of eosinophils and bronchial epithelium cells; observed an increase in the release of inflammatory cytokines (NF-KB, AP-1, MAPK, IL-1, IL-6, TNF-alpha) [23] and in the expression of adhesion molecules, such as ICAM-1, in the co-culture systems. In our cellular model of human nasal epithelial cells, we have confirmed the elevation of the levels of pro-inflammatory cytokines of epithelial origin and alarmines such as IL-6, IL-8, IL-1 $\beta$, as well as the pro-inflammatory chemokine CCL2 associated with Th2 response and monocyte chemotactic, as well as the pro-inflammatory chemokine CXCL1 potent chemotactic for neutrophils. 
Bronchial epithelial cells act as immunoregulators in allergic respiratory diseases through the expression of adhesion molecules on the cell surface, such as ICAM [24,25]. We have also confirmed the HDM-induced expression of VEGF (vascular endothelial growth factor) and ICAM1 (intercellular adhesion molecule of endothelial and immune cells). Although the increase in angiogenesis is a characteristic feature of the remodeling of the asthmatic airways as a consequence of inflammation, its activation seems to be confirmed from the beginning of exposure to the allergen, associated with an expression of ICAM-1 molecules that will favor the adhesion of inflammatory effector cells.

IL-10 is expressed in the nasal mucosa of allergic patients to HDM both in the epithelium and in the blood vessel endothelial cells, playing a role in the pathogenesis of allergic rhinitis as an immunosuppressive cytosine and revealed by Muller et al. [26] after nasal challenges with allergens and subsequent biopsies. Similarly, in our experimental model, we have observed a marked decrease in the production of IL-10 (an anti-inflammatory cytokine) after induction with LPS, Poly (I: C) and house dust mites.

On the other hand, autophagy has been shown to play an essential role in the lung inflammatory response induced by infection and stress. According to Racanelli et al [27], autophagy seems to play an important role in the pathogenesis of asthma. High levels of autophagy have been seen in sputum granulocytes, peripheral blood cells, and eosinophils from patients with asthma, although little is known about their role in allergic rhinitis. Also, genetic mutations in autophagy genes have been associated with asthma; for example, polymorphisms in ATG5 correlate with airway remodeling and loss of lung function in asthma [28]. Environmental pollutants and allergens can induce autophagy, and autophagy has been suggested to be essential for inhibiting spontaneous lung inflammation and for secretion of airway mucus by goblet cells. Mice with autophagy deficiency (Atg5/- and Atg7-/) develop spontaneous sterile lung inflammation [29]. Cell culture experimental model systems suggest that, in the lung epithelium, activation of autophagy may be deleterious inducing disease progression and promote the Th2 epithelial response in asthma. Our data also show that HDM triggered the expression of several key genes in the nasal epithelium, including autophagy, unfolded protein response (UPR), matrix metalloproteinases (MMP), senescence, and inflammation16. HDM activates the autophagy genes: ATG3, ATG5, ATG7, BECLIN-1 and P62. These data support other previous studies in other respiratory diseases, as well as in chronic obstructive pulmonary disease (COPD) $[27,30]$.

Endoplasmic reticulum (ER) stress and the unfolded protein response (UPR) pathway may play a fundamental role in the regulation of epithelium-dendritic cell interaction, which is crucial for the initiation and amplification of the allergic response [21]. The accumulation of misfolded proteins in the ER can be signaled through IRE1 $\alpha$ (transmembrane receptor that includes the enzyme $1 \alpha$ that requires inositol), ATF6 (activation of transcription factor 6) and PERK (double-stranded RNA-dependent protein kinase PKR ER kinase). If the first two signaling pathways fail to restore ER stress initiated by the GRP78 chaperone, homologous protein C / EBP (CHOP) will mediate apoptosis using PERK signaling. In our cell model, treatment with HDM also increases the expression of UPR, including the ATF6, CHOP and IRE1 genes, which are critical in allergic reactions and in asthmatic patients1 [21,31].

It has been described that cellular senescence is characterized by irreversible cell cycle arrest, leading to an increase in pro-inflammatory mediators, less capacity for tissue regeneration or repair, greater accumulation of senescent cells [32], being an important risk factor in the lung for the development of the asthma [33]. Among the studies that relate cell senescence and asthma, it is worth mentioning the research performed by $\mathrm{Wu}$ et al. [34] who observed cellular senescence induced by TSLP (Thymic Stromal Lymphopoietin) with elevated levels of P21 and P16 in human epithelial cells, being essential for the remodeling of the airways in vitro; as well as the study with a plasminogen activator inhibitor (PAI-1), mediator of cell senescence and fibrosis, which could activate p53 and mediate the senescence of type II alveolar cells (ATII) induced by bleomycin and doxoru- 
bicin [35]. Therefore, senescence is triggered by factors such as aging, DNA damage, oxidative stress, mitochondrial dysfunction [16]. In order to study the activation of the cell senescence pathways we can use different markers: p53-ARF pathway (crucial mediator which plays a role in the cell responses to DNA damage) and the p16-pRb pathway (induced by a variety of stressful stimuli). In our results, the inflammatory genes IL-1a, IL$1 \mathrm{~b}$, and IL- 6 are also activated, and finally, HDM also triggered senescence genes, including Cdkn1a (p21), Cdkn2a (p16), and Cdkn1b (p27) but not p53.

Elovanoids have been recently studied in an experimental model of retinal cells showing protection against neurodegenerative diseases such as age-related macular degeneration (AMD) and Alzheimer's, promoting repair, remodeling and regeneration [12]. In this study, ELV-34 was shown to play a relevant role in promoting cell viability and decreasing cytotoxicity after HNEpC sensitization to HDM. Furthermore, our results indicate that the levels of IL-6, IL-1 $\beta$, IL-8, VEGF, ICAM1, CXCL1, and CCL2 that had increased with HDM sensitization, were significantly reduced with ELV-34 treatment. Likewise, the level of IL-10 was restored by treatment with ELV34. Therefore, ELV-34 manages to decrease the production of pro-inflammatory cytokines, increasing the production of anti-inflammatory cytokines and decreasing the expression of adhesion molecules and vascular endothelial growth factor after exposure to inflammatory inducers and the HDM allergen.

Liu et al. [36], reviewing the role of autophagy in allergic inflammation, observed that inhibition of autophagy in a murine OVA asthma model reduces airway response, eosinophilia, and inflammation. Interestingly, treatment with ELV-34 decreases the induction of transcription of these genes. Furthermore, $\beta$-galactosidase staining, which is a marker of the senescence-associated secretory phenotype (SASP), was also performed on HDMchallenged nasal epithelial cells with or without ELV-34 [37]. Treatment with ELV-34 showed a marked decrease in $\beta$-galactosidase compared to cells treated with HDM alone.

In summary, our results shows that ELV-34 promotes cell viability and sequesters cytotoxicity upon HDM sensitization of HNEpC. Also, the levels of IL-6, IL-1 $\beta$, IL-8, VEGF, ICAM1, CXCL1 and CCL2 increased upon HDM sensitization, which was reduced significantly upon ELV-34 treatment. IL-10 level was reduced upon HDM sensitization, which was restored by ELV34 treatment. Our data also shows that HDM triggered the expression of several key genes in nasal epithelium, including autophagy, unfolded protein response (UPR), Matrix metalloproteinases (MMPs), senescence and inflammation [16]. HDM activates the autophagy genes: ATG3, ATG5, ATG7, BECLIN-1 and P62. This data supports other previous studies in other respiratory diseases as well as in chronic obstructive pulmonary disease (COPD) [27,30]. HDM treatment also increase the expression of UPR, including Atf6, Chop and Ire1 genes, which are critical in allergic reactions and asthmatic patient $[21,31,38]$.

In addition, the inflammatory genes, IL- $1 \alpha$, IL- $1 \beta$ and IL- 6 , are activated as well and finally, the HDM also triggered the senescence genes, including Cdkn1a (p21), Cdkn2a (p16) and Cdkn1b (p27) but not p53. Interestingly, ELV-34 treatment diminishes the induction of the transcription of these genes. Moreover, $\beta$-galactosidase staining which is a marker of senescence associated secretory phenotype (SASP) was also performed in the nasal epithelial cells challenged with HDM with or without ELV-34 [37]. ELV-34 treatment showed marked decrease in $\beta$-galactosidase when compared with cells treated with HDM alone.

Although more studies are needed in this regard, our results show that ELV-34 is capable of inhibiting cytokines derived from epithelial cells, reducing the activation signal of the innate immune system by HDM, necessary to maintain adaptive immunity, restore the epithelial mucosal barrier and reduce the transcription of senescence and autophagy genes, being able to constitute a therapeutic option in the prevention and treatment of allergy to HDM.

Allergens are associated with the SASP program, the inflammaging phenomenon and senescent cells as a consequence of immuno-inflammatory dysfunction that leads to 
increases in oxidative stress, mitochondrial dysfunction and the secretion of cytokines that contribute to cellular damage and chronic inflammation.

Elovanoids may represent a new therapeutic as shown by the results provided in relation to their activity on SASP, inflammaging, matrix remolding, autophagia and accumulation of senescent cells, restoring immune-inflammatory dysfunction and regulating homeostasis in the prevention and progression of allergic pathologies.

\section{Materials and Methods}

\subsection{Experimental design}

The experimental design (Figure 1) with primary human nasal epithelial cells (HNEpC) allowed to assess the prohomeostatic protective signaling of ELV32 and ELV34 in relation to cytotoxicity, cell viability, cytokine production and expression of genes involved in inflammatory and senescence processes. HNEpC were challenged with different stressors (LPS, poly(I:C) or house dust mite extract and, 30 minutes later, the cells were treated with ELV32 or ELV34 (500nM). Cells were analyzed for cytotoxicity and cell viability and also cell supernatants were collected at 24 hours in order to assay the expression of pro-inflammatory cytokines and chemokines.

\subsection{Cell line}

Cryopreserved human nasal epithelial cells HNEpC were purchased from PromoCell GmbH, Heidelberg, Germany. (Catalog\# C-1260, Lot\# 436Z028). These cells are primary nasal epithelial cells obtained from the nasal mucosa of a 50 year old Caucasian male. HNEpC were grown to 80\% confluence in Promocell's Airway epithelial cell growth medium (Catalog\# C-21060) which was supplemented with airway epithelial cell growth medium supplement pack (Catalog\# C-39160) and Penicillin/Streptomycin.

\subsection{Cell inductors}

HNEpC were challenged using several stressors: House Dust Mite extract from Dermatophagoides pteronyssinus (D.P.) (Catalog\# 3033) lyophilized extract were obtained from Chondrex, Inc. and used at $30 \mu \mathrm{g} / \mathrm{mL}$ to challenge HNEpC. House Dust Mite extract from Dermatophagoides farinae (D.F.) (Catalog\# 3040) lyophilized extract were obtained from Chondrex, Inc. D.F. and used at $30 \mu \mathrm{g} / \mathrm{mL}$ to challenge HNEpC. House Dust Mite extract - HDM a mixture of both (D.P.) \& (D.F.) was used at $(15 \mu \mathrm{g} / \mathrm{mL}+15 \mu \mathrm{g} / \mathrm{mL})$ to challenge HNEpC.

Lipopolysaccharide (LPS) from Escherichia coli serotype 0111:B4 (Sigma-Aldrich Catalog\# L4391). LPS is the principal component of Gram-negative bacteria that activates the innate immune system through its recognition by Toll-like receptor 4 (TLR4). This leads to a signaling cascade that ultimately results in the activation of NF- $\mathrm{kB}$ and the production of proinflammatory cytokines. LPS we used for our experiments is a preparation of smooth (S)-form LPS purified from the Gram-negative E. coli 0111:B4 that was used at $30 \mu \mathrm{g} / \mathrm{mL}$ to challenge HNEpC.

Polyinosinic-polycytidylic acid (poly(I:C) or poly(rI):poly(rC)) is a synthetic analog of double-stranded viral RNA (dsRNA), a molecular pattern associated with viral infection such as loss of epithelial integrity, increased production of mucus and inflammatory cytokines. Poly(I:C), a TLR3 agonist activates the antiviral pattern recognition receptors TLR3, RIG-I/MDA5 and PKR, thereby inducing signaling via multiple inflammatory pathways, including NF- $\kappa$ B and IRF. High Molecular Weight Poly(I:C) comprises long strands of inosine poly(I) homopolymer annealed to strands of cytidine poly $(\mathrm{C})$ homopolymer. 
The average size of Poly(I:C) HMW is from $1.5 \mathrm{~kb}$ to $8 \mathrm{~kb}$. Poly(I:C) (Catalog\# P1530) were obtained from Sigma-Aldrich and used at $100 \mu \mathrm{g} / \mathrm{mL}$ to challenge HNEpC.

\subsection{Cell survival}

Cell viability was analyzed using PrestoBlue HS (ThermoFisher Scientific) reagent (Cata$\log \#$ C50201, Invitrogen). The PrestoBlue HS Cell Viability Reagent is a complete add and read, nontoxic reagent that does not require cell lysis. The highly purified resazurin that is used for PrestoBlue HS results in a reagent with a $>50 \%$ decrease in background fluorescence and $a>100 \%$ increase in the signal to background ratio.

On entering live cells, the cellular reducing environment reduces resazurin to resorufin a compound that is red and highly fluorescent. Viable cells continuously convert resazurin to resorufin increasing the overall fluorescence and color of the media surrounding the cells. Also, the conversion of resazurin to resorufin results in a pronounced color change, therefore cell viability can be detected using absorbance-based plate readers. Fluorescence is read using a fluorescence excitation wavelength of $560 \mathrm{~nm}$ (excitation range is 540-570 $\mathrm{nm}$ ) and an emission of $590 \mathrm{~nm}$ (emission range is 580-610-nm).

\subsection{Cytotoxicity analysis}

Cytotoxicity was measured using CyQuant LDH assay kit (Catalog\# C20301, ThermoFisher Scientific) what evaluates damage to the plasma membrane releases LDH into the surrounding cell culture media. The extracellular LDH in the media can be quantified by a coupled enzymatic reaction in which LDH catalyzes the conversion of lactate to pyruvate via NAD+ reduction to NADH. Oxidation of NADH by diaphorase leads to the reduction of a tetrazolium salt (INT) to a red formazan product that can be measured spectrophotometrically at $490 \mathrm{~nm}$. The level of formazan formation is directly proportional to the amount of $\mathrm{LDH}$ released into the medium, which is indicative of cytotoxicity.

\subsection{Pro-inflammatory cytokine profile analysis}

In addition, the expression of the following cytokines were analyzed in the supernant of HNEpC exposed to different allergic inductors: IL-6 (Catalog\# 6802, Chondrex), IL-1 $\beta$ (Catalog\# 6805, Chondrex), IL-8/CXCL8 (Quantikine ELISA Kit, Catalog\# D8000C, R\&D Systems), VEGF (Catalog\# 6810, Chondrex), ICAM1(CD54) (ELISA Kit, Catalog\# ab100640, Abcam), CXCL1/KC/GRO (Catalog\# 6825, Chondrex) and CCL2/MCP-1 (Cata$\log \# 6821$, Chondrex), and IL-10 (Catalog\# 6806, Chondrex).

\subsection{Cellular Senescence Assay ( $\beta$-galactosidase expression)}

Cellular senescence was measured in HNEpC by analyzing the expression of beta-galatosidase using a beta-gal staining kit (Spider $\beta$-gal Kit, Catalog\#SG04, Dojindo). SPiDER$\beta \mathrm{Gal}$ allows to detect senescence-associated (SA)- $\beta$-gal with high sensitivity being a reagent which possesses a high cell-permeability and a high retentivity inside cells. SA- $\beta$-gal can be detected specifically not only in living cells but also fixed cells by using a reagent (Bafilomycin A1) to inhibit endogenous $\beta$-galactosidase activity.

\subsection{Gene expression}

Total RNA was isolated using RNeasy Plus Mini Kit (Qiagen). Quantitative PCR was performed in a CFX-384 Real-Time PCR system (Bio-Rad). The expression of target genes was normalized to the geometric mean of housekeeping genes and relative expression was calculated by the comparative threshold cycle method $(\Delta \Delta \mathrm{CT})$. Table 1 shows a list of the primers used in the study. 
Table 1. List of primers

\begin{tabular}{|c|c|c|c|}
\hline Protein & $\begin{array}{l}\text { Gene } \\
\text { Name }\end{array}$ & Forward Sequence & Reverse Sequence \\
\hline ATG3 & Atg3 & GATGGCGGATGGGTAGATACA & TCTTCACATAGTGCTGAGCAATC \\
\hline ATG5 & Atg5 & AGAAGCTGTTTCGTCCTGTGG & AGGTGTTTCCAACATTGGCTC \\
\hline ATG7 & Atg7 & ATGATCCCTGTAACTTAGCCCA & CACGGAAGCAAACAACTTCAAC \\
\hline Beclin-1 & Becn1 & GGTGTCTCTCGCAGATTCATC & TCAGTCTTCGGCTGAGGTTCT \\
\hline p62 & Nup62 & CСТTCCAACCAGCCACAAGTAC & CGAAGCAAGAGTCGCTGTTCCA \\
\hline ATF6 & Atf6 & GACTCACCCATCCGAGTTGTG & CTCCCAGTCTTCATCTGGTCC \\
\hline CHOP & Aff4 & CTGGAAGCCTGGTATGAGGAT & CAGGGTCAAGAGTAGTGAAGGT \\
\hline IRE1 & Ern1 & CCGAACGTGATCCGCTACTTCT & CGCAAAGTCCTTCTGCTCCACA \\
\hline MMP8 & Mmp8 & AАTCCTTGCCCATGCСTTTCAACC & CCAAATTCATGAGCAGCCACGAGA \\
\hline MMP2 & Mmp2 & CCTTAAAAGTATGGAGCGACGTCA & AGCGTTCCCATACTTTACGCG \\
\hline MMP9 & Mmp9 & ACGACATAGACGGCATCCAGTATC & AGGTATAGTGGGACACATAGTGGG \\
\hline MMP3 & Mmp3 & ACCAACCTATTCCTGGTTGCTGCT & ATGGAAACGGGACAAGTCTGTGGA \\
\hline MMP12 & Mmp12 & TAGAAGCAACTGGGCAACTGGACA & ACCGCTTCATCCATCTTGACСTCT \\
\hline TIMP1 & Tipm1 & GGAGAGTGTCTGCGGATACTTC & GCAGGTAGTGATGTGCAAGAGTC \\
\hline TIMP2 & Tipm2 & ACССTCTGTGACTTCATCGTGC & GGAGATGTAGCACGGGATCATG \\
\hline $\mathrm{p} 21^{\mathrm{CIP}}$ & $C D K N 1 A$ & TGTCCGTCAGAACCCATGC & AAAGTCGAAGTTCCATCGCTC \\
\hline p16 ${ }^{\text {INK4a }}$ & $C D K N 2 A$ & GGGGGCACCAGAGGCAGT & GGTTGTGGCGGGGGCAGTT \\
\hline p27KIP & $C D K N 1 B$ & TAATTGGGGCTCCGGCTAACT & TGCAGGTCGCTTCCTTATTCC \\
\hline p53 & TRP53 & CCССТCСTGGCCCСТGTCATCTTC & GCAGCGCCTCACAACCTCCGTCAT \\
\hline $\mathrm{IL}-1 \alpha$ & $I L-1 \alpha$ & TGTATGTGACTGCCCAAGATGAAG & AGAGGAGGTTGGTCTCACTACC \\
\hline IL-6 & $I L-6$ & CTGCAAGAGACTTCCATCCAG & AGTGGTATAGACAGGTCTGTTGG \\
\hline IL-1 $\beta$ & $I L-1 \beta$ & AGCTACGAATCTCCGACCAC & CGTTATCCCATGTGTCGAAGAA \\
\hline $\mathrm{ACTB}$ & $A c t B$ & AGCCTCGCCTTTGCCGA & CTGGTGCCTGGGGCG \\
\hline GAPDH & GAPDH & TGGACCTGACCTGCCGTCTA & CCCTGTTGCTGTAGCCAAATTC \\
\hline
\end{tabular}

Author Contributions: Conceptualization, R.P. and N.B.; methodology, D.R., S.B., A.R., M.B. and R.A.; formal analysis, D.R., A.R., M.B. and R.A.; investigation, D.R., A.R., M.B. and R.A.; resources, R.P. and N.B.; data curation, D.R., A.R., M.B.; writing-original draft preparation, A.R., M.B. and R.P; writing - review and editing, A.R., M.B., S.B., R.P. and N.B; project administration, R.P and N.B.; funding acquisition, R.P and N.B. All authors have read and agreed to the published version of the manuscript.

Funding: Part of this project has been possible thanks to the financing obtained through the OTRI 2020907094 contract signed between UPNA and Trim Biotech S.L.

Acknowledgments: The authors are especially grateful to Dr. Bhattacharjee for his contribution in data processing together with Drs. Resano and Barajas.

Conflicts of Interest: Ricardo Palacios (CEO) and David Rodríguez (Research Department Director) work at Diater Laboratorio de Diagnórica y Aplicaciones Teraprapeuticas S.A and declare that they had no involved in the final decision to publish the results neither in the orientation of the results obtained in the present study. The other authors declare no conflict of interest. 
1. Calderón, M. A., Linneberg, A., Kleine-Tebbe, J., De Blay, F., Hernandez Fernandez de Rojas, D., Virchow, J. C., \& Demoly, P. (2015). Respiratory allergy caused by house dust mites: What do we really know? Journal of Allergy and Clinical Immunology, 136(1), 38-48. https://doi.org/10.1016/j.jaci.2014.10.012

2. Dumez, M.-E., Herman, J., Campizi, V., Galleni, M., Jacquet, A., \& Chevigné, A. (2014). Orchestration of an uncommon maturation cascade of the house dust mite protease allergen quartet. Frontiers in Immunology, 5, 138. https://doi.org/10.3389/fimmu.2014.00138

3. Gandhi, V. D., Davidson, C., Asaduzzaman, M., Nahirney, D., \& Vliagoftis, H. (2013). House Dust Mite Interactions with Airway Epithelium: Role in Allergic Airway Inflammation. Current Allergy and Asthma Reports, 13(3), 262-270. https://doi.org/10.1007/s11882-013-0349-9

4. Gregory, L. G., \& Lloyd, C. M. (2011). Orchestrating house dust mite-associated allergy in the lung. Trends in Immunology, 32(9), 402-411. https://doi.org/10.1016/j.it.2011.06.006

5. Jacquet, A. (2011a). The Role of the House Dust Mite-Induced Innate Immunity in Development of Allergic Response. International Archives of Allergy and Immunology, 155(2), 95-105. https://doi.org/10.1159/000320375

6. Jacquet, A. (2011b). The role of innate immunity activation in house dust mite allergy. Trends in Molecular Medicine, 17(10), 604-611. https://doi.org/10.1016/j.molmed.2011.05.014

7. Piyadasa, H., Altieri, A., Basu, S., Schwartz, J., Halayko, A. J., \& Mookherjee, N. (2016). Biosignature for airway inflammation in a house dust mite-challenged murine model of allergic asthma. Biology Open, 5(2), 112-121. https://doi.org/10.1242/bio.014464

8. Herman, J., Thelen, N., Smargiasso, N., Mailleux, A.-C., Luxen, A., Cloes, M., De Pauw, E., Chevigné, A., Galleni, M., \& Dumez, M.-E. (2014). Der $p 1$ is the primary activator of Der $p$ 3, Der $p 6$ and Der $p 9$ the proteolytic allergens produced by the house dust mite Dermatophagoides pteronyssinus. Biochimica et Biophysica Acta (BBA) - General Subjects, 1840(3), 1117-1124. https://doi.org/10.1016/j.bbagen.2013.11.017

9. Woo, L. N., Guo, W. Y., Wang, X., Young, A., Salehi, S., Hin, A., Zhang, Y., Scott, J. A., \& Chow, C. W. (2018). A 4-Week Model of House Dust Mite (HDM) Induced Allergic Airways Inflammation with Airway Remodeling. Scientific Reports, 8(1), 6925. https://doi.org/10.1038/s41598-018-24574-x

10. Bazan, N. G. (2018). Docosanoids and elovanoids from omega-3 fatty acids are pro-homeostatic modulators of inflammatory responses, cell damage and neuroprotection. Molecular Aspects of Medicine, 64, 18-33. https://doi.org/10.1016/j.mam.2018.09.003

11. Bhattacharjee, S., Jun, B., Belayev, L., Heap, J., Kautzmann, M.-A., Obenaus, A., Menghani, H., Marcell, S. J., Khoutorova, L., Yang, R., Petasis, N. A., \& Bazan, N. G. (2017). Elovanoids are a novel class of homeostatic lipid mediators that protect neural cell integrity upon injury. Science Advances, 3(9), e1700735. https://doi.org/10.1126/sciadv.1700735

12. Do, K. V., Kautzmann, M.-A. I., Jun, B., Gordon, W. C., Nshimiyimana, R., Yang, R., Petasis, N. A., \& Bazan, N. G. (2019). Elovanoids counteract oligomeric $\beta$-amyloid-induced gene expression and protect photoreceptors. Proceedings of the National Academy of Sciences of the United States of America, 116(48), 24317-24325. https://doi.org/10.1073/pnas.1912959116

13. Jun, B., Mukherjee, P. K., Asatryan, A., Kautzmann, M.-A., Heap, J., Gordon, W. C., Bhattacharjee, S., Yang, R., Petasis, N. A., \& Bazan, N. G. (2017). Elovanoids are novel cell-specific lipid mediators necessary for neuroprotective signaling for photoreceptor cell integrity. Scientific Reports, 7(1), 5279. https://doi.org/10.1038/s41598-017-05433-7

14. Bazan, N. G. (2020). Overview of how N32 and N34 elovanoids sustain sight by protecting retinal pigment epithelial cells and photoreceptors. Journal of Lipid Research. https://doi.org/10.1194/jlr.TR120001137

15. Nettleton, JA., Salem, N. (2019). International Society for the Study of Fatty Acids and Lipids 2018 Symposium: Arachidonic and Docosahexaenoic Acids in Infant Development. Ann Nutr Metab, 74:83-91. https://doi:10.1159/000495906

16. Sachdeva, K., Do, D. C., Zhang, Y., Hu, X., Chen, J., \& Gao, P. (2019). Environmental Exposures and Asthma Development: Autophagy, Mitophagy, and Cellular Senescence. Frontiers in Immunology, $10,2787$. https://doi.org/10.3389/fimmu.2019.02787

17. Yong-Chen, L., Wen-Chen, Y., Pamela, S.O. Review Article LPS/TLR4 signal transduction pathway. Cytokine 42 (2008) 145151. https://doi.org/10.1016/j.cyto.2008.01.006

18. Alexopoulou L, Holt AC, Medzhitov R, Flavell RA. Recognition of double-stranded RNA and activation of NF- $\kappa B$ by Tolllike receptor 3. Nature. 2001;413(6857):732-738. [PubMed] [Google Scholar]

19. Schulz O, Sewell HF, Shakib F. Proteolytic cleavage of CD25, the alpha subunit of the human T cell interleukin 2 receptor, by Der p 1, a major mite allergen with cysteine protease activity. J Exp Med. 1998 Jan 19;187(2):271-5. doi: 10.1084/jem.187.2.271. PMID: 9432986; PMCID: PMC2212095.

20. Ghaemmaghami AM, Gough L, Sewell HF, Shakib F. The proteolytic activity of the major dust mite allergen Der $\mathrm{p} 1$ conditions dendritic cells to produce less interleukin-12: allergen-induced Th2 bias determined at the dendritic cell level. Clin Exp Allergy. 2002 Oct;32(10):1468-75. doi: 10.1046/j.1365-2745.2002.01504.x. PMID: 12372127.

21. Jeong, J. S., Kim, S. R., Cho, S. H., \& Lee, Y. C. (2017). Endoplasmic Reticulum Stress and Allergic Diseases. Current Allergy and Asthma Reports, 17(12), 82. https://doi.org/10.1007/s11882-017-0751-9

22. Wong CK, Li ML, Wang CB, Ip WK, Tian YP, Lam CW. House dust mite allergen Der p 1 elevates the release of inflammatory cytokines and expression of adhesion molecules in co-culture of human eosinophils and bronchial epithelial cells. Int Immunol. 2006 Aug;18(8):1327-35. doi: 10.1093/intimm/dx1065. Epub 2006 Jun 23. PMID: 16798840.

23. King C, Brennan S, Thompson PJ, Stewart GA. Dust mite proteolytic allergens induce cytokine release from cultured airway epithelium. J Immunol. 1998 Oct 1;161(7):3645-51. PMID: 9759888. 
24. Pawankar, R. 2002. Epithelial cells as immunoregulators in allergic airway diseases. Curr. Opin. Allergy Clin. Immunol. 2:1.

25. Atsuta, J., Plitt, J., Bochner, B. S. and Schleimer, R. P. 1999. Inhibition of VCAM-1 expression in human bronchial epithelial cells by glucocorticoids. Am. J. Respir. Cell Mol. Biol. 20:643.

26. Muller, B. Interleukin-10 In Nasal Mucosa Protects Against Allergic Symptoms. Journal of Allergy and Clinical Immunology. Volume 119, Issue 2, February 2007, Page 525.

27. Racanelli, A. C., Kikkers, S. A., Choi, A. M. K., \& Cloonan, S. M. (2018). Autophagy and inflammation in chronic respiratory disease. Autophagy, 14(2), 221-232. https://doi.org/10.1080/15548627.2017.1389823

28. Martin LJ, Gupta J, Jyothula SS, Butsch Kovacic M, Biagini Myers JM, Patterson TL, Ericksen MB, He H, Gibson AM, Baye TM, et al. Functional variant in the autophagy-related 5 gene promotor is associated with childhood asthma. PloS One. 2012;7(4):e33454. doi:10.1371/journal.pone.0033454. PMID:22536318

29. Abdel Fattah E, Bhattacharya A, Herron A, Safdar Z, Eissa NT. Critical role for IL-18 in spontaneous lung inflammation caused by autophagy deficiency. J Immunol. (2015) 194:5407-16. doi: 10.4049/jimmunol.1402277

30. Ryter, S. W., Lee, S.-J., \& Choi, A. M. (2010). Autophagy in cigarette smoke-induced chronic obstructive pulmonary disease. Expert Review of Respiratory Medicine, 4(5), 573-584. https://doi.org/10.1586/ers.10.61

31. Pathinayake, P. S., Hsu, A. C.-Y., Waters, D. W., Hansbro, P. M., Wood, L. G., \& Wark, P. A. B. (2018). Understanding the Unfolded Protein Response in the Pathogenesis of Asthma. Frontiers in Immunology, 9. https://doi.org/10.3389/fimmu.2018.00175

32. Golomb L, Sagiv A, Pateras IS, Maly A, Krizhanovsky V, Gorgoulis V, et al. AgeRassociated inflammation connects RASRinduced senescence to stem cell dysfunction and epidermal malignancy.Cell Death and Differentiation. 2015; 22: 1764-1774;doi:10.1038/cdd.2015.21

33. Fedorov IA, Wilson SJ, Davies DE, Holgate ST. Epithelial stress and structural remodelling in childhood asthma. Thorax. (2005) 60:389-94. doi: 10.1136/thx.2004.030262

34. Wu J, Dong F, Wang RA, Wang J, Zhao J, Yang M, et al. Central role of cellular senescence in TSLP-induced airway remodeling in asthma. PLoS ONE. (2013) 8:e77795. doi: 10.1371/journal.pone.0077795

35. Jiang C, Liu G, Luckhardt T, Antony V, Zhou Y, Carter AB, et al. Serpine 1 induces alveolar type II cell senescence through activating p53- p21-Rb pathway in fibrotic lung disease. Aging Cell. (2017) 16:1114-24. doi: 10.1111/acel.12643

36. Liu JN, Suh DH, Trinh HK, Chwae YJ, Park HS, Shin YS. The role of autophagy in allergic inflammation: A new target for severe asthma. Experimental \& Molecular Medicine. 2016;48(7):e243. doi:10.1038/ emm.2016.38.

37. Mohamad Kamal, N. S., Safuan, S., Shamsuddin, S., \& Foroozandeh, P. (2020). Aging of the cells: Insight into cellular senescence and detection Methods. European Journal of Cell Biology, 99(6), 151108. https://doi.org/10.1016/j.ejcb.2020.151108

38. Abu Khweek, A., Kim, E., Joldrichsen, M. R., Amer, A. O., \& Boyaka, P. N. (2020). Insights Into Mucosal Innate Immune Responses in House Dust Mite-Mediated Allergic Asthma. Frontiers in Immunology, $11,534501$. https://doi.org/10.3389/fimmu.2020.534501 